\title{
Age-associated memory impairment and related disorders
}

\author{
J. T. O’Brien
}

Complaints of poor memory are common in the healthy elderly and to many it may seem unsurprising that cognitive function declines with 'normal' ageing. Virtually every biological system alters with age and, just as a 70-year-old cannot run as fast or hear as well as when he or she was 20 , it is perhaps inevitable that cognitive function also becomes impaired. However, far from being straightforward, the nature, classification and clinical significance of age-related cognitive changes that fall short of dementia remain a most controversial and difficult area (see O'Brien \& Beats, 1994; Dal Forno \& Kawas, 1995). The recent emergence of new drugs for the treatment of Alzheimer's disease (donepezil and rivastigmine) and related disorders has emphasised the need to study groups with milder degrees of cognitive impairment. It is necessary to determine whether such conditions are benign and nonprogressive, or harbingers of progressive dementia and so appropriate conditions to target for early therapeutic intervention. The presence of age-related cognitive changes raises other important issues including why such changes occur, how they should be classified and whether, even if 'benign', they can and should be treated.

\section{Cognitive changes associated with normal ageing}

If groups of young and elderly volunteers are compared on various cognitive tests then, on average, the elderly perform significantly less well. For example, norms on the Wechlser Adult
Intelligence Scale (WAIS) decline by up to $30 \%$ between the ages of 20 and 75 (Sattler, 1982). Performance items are affected to a greater extent than verbal abilities, although even verbal components decline significantly. Early studies of agerelated changes presented a rather gloomy view of cognitive decline with age, suggesting that peak ability was reached around the age of 25 with a relentless decline thereafter (Botwinick, 1977). However, such conclusions were largely based on cross-sectional studies which had numerous methodological difficulties including inadequate matching and the inability to take into account cohort-specific factors such as education, familiarity with technology and tests, health, nutrition and life experiences. Longitudinal and cross-sequential studies (which sample several different age cohorts at different time points) can account for cohortspecific factors and have played a crucial role in confirming that significant cognitive decline does occur with age - although they demonstrate that decline generally begins much later in life than previously thought, with most tests remaining relatively stable until the age of 60 (Schaie, 1983; Schaie et al, 1998).

Not all cognitive abilities are equally affected by ageing. Cognitive function can be characterised in a number of ways, although the division into crystallised and fluid intelligence is often made (Horn \& Cattell, 1967). Fluid intelligence reflects the ability to acquire and manipulate new information and is measured by tasks involving problemsolving and speed of performance. Crystallised intelligence is the 'cumulative end product' of information acquired as a result of fluid intelligence and is measured using vocabulary and information

John O'Brien is Senior Lecturer in Old Age Psychiatry at the Wolfson Research Centre, Newcastle General Hospital, Westgate Road, Newcastle-upon-Tyne, NE4 6BE. His research interests include the early diagnosis of dementia, particularly dementia with Lewy bodies, the use of neuroimaging in old age psychiatry, and neurobiological changes in late-life depression. 
tests and tests of knowledge. Decline in fluid intelligence can be clearly demonstrated with age, whereas crystallised intelligence (as measured by vocabulary) accumulates from early adulthood, stabilises in middle life and does not decline in later life, except perhaps at the extremes (Schaie et al, 1998). There is also a differential effect of ageing on memory tasks. A clinical division of memory is often made between long-term and short-term memory, although other divisions have been suggested such as that between episodic (remembering events) and semantic (general knowledge of the world) memory and procedural and declarative memory. Procedural memory is memory not requiring conscious recollection of experience and includes skill-based learning (e.g. mirror drawing) and priming. Declarative memory requires conscious recall and is the form of memory classically tested clinically (e.g. recall of a name and address). Different neuroanatomical substrates may underlie these different types of memory, for example declarative and episodic memory are very dependent on medial temporal lobe regions such as the hippocampus, while semantic memory may depend more on the temporal cortex, and procedural memory on the basal ganglia. Declarative and episodic memory show greater decline with age than semantic and procedural memory.

Rather than assess cognition in terms of the specific processes involved, some have postulated that ageing effects are more general. For example, information-processing is significantly slower in older subjects (Birren et al, 1979) and this may affect ability to encode efficiently, as well as speed of retrieval. Craik \& Byrd (1982) suggested that the elderly may have a smaller pool of processing resources available and that these resources are unable to cope with task demands or, alternatively, that the pool of processing resources remains the same but the demand on this pool increases with age, leaving less available for other tasks.

One important principle which emerges with most studies of ageing, whether they be biological or psychological, is that there is increasing variability in performance between subjects with advancing age (Nelson \& Dannefer, 1992). This results in mean changes in cognitive performance, the primary end-point of most studies, being more difficult to interpret because some older individuals change very little while others deteriorate dramatically. An increase in variability could be explained by contamination of results with the inadvertent inclusion of those with early dementia, but the finding that such variability with ageing is a feature of biological as well as cognitive measures suggests that this is not the whole explanation. Neuropsychological testing of a 118-year-old French lady who had the performance of an 80-year-old, suggested that while some cognitive decline is likely to occur even in the 'super-fit', global decline is not inevitable even at the extremes of human longevity (Ritchie, 1995).

\section{Neurobiological basis of age-related cognitive changes}

Brain weight is known to decrease with age and total mass declines by up $20 \%$ by the age of 90 . Initially, it was thought that this weight loss reflected loss of cortical neurons and the classic study by Brody (1955) showed extensive loss in all cortical lobes, a finding subsequently confirmed by others (Anderson et al, 1983). However, these studies suffered from methodological problems including not controlling for cranial capacity or brain shrinkage during post-mortem. Cortical neuronal loss may not be as large as previously supposed (Terry $e t$ al, 1987), although there is evidence of age-related neuronal loss in other areas such as the hippocampus, locus coeruleus, substantia nigra and nucleus basalis of Meynert. Other brain changes with age include increased accumulation of lipofuscin, granulovacuolar degeneration, a reduction in dendritic branching and synaptic density and the appearance of plaques and tangles (Tomlinson \& Henderson, 1976). In normal ageing neurofibrillary tangles are usually limited to the hippocampus, in contrast to Alzheimer's disease where they are widespread and present in greater densities (Tomlinson \& Henderson, 1976). Although most develop at least some tangles, about $25 \%$ of those who have reached the age of 90 will be free from plaques. However, as well as degenerative processes with ageing there is some evidence that compensatory mechanisms may exist, for example, agerelated increases in pyramidal cell dendritic spine density have been described (Buell \& Coleman, 1979).

Although in dementia the relationship between brain changes and cognitive impairment has been widely studied, the neurobiological correlates of agerelated cognitive changes are unclear. Mild cognitive impairment has been correlated with neurofibrillary tangles in the entorhinal cortex (Hof et al, 1996) and the hippocampus (Giannakopoulos et al, 1997), which would be consistent with the greater effects of ageing on episodic and declarative memory.

Neuroimaging studies have yielded mixed results regarding correlations between brain changes and cognitive decline that fall short of dementia. For example, Laakso et al (1998) found hippocampal 
volume on magnetic resonance imaging to be unchanged in subjects with age-associated memory impairment (AAMI), whereas Parnetti et al (1996) suggested subjects with AAMI had magnetic resonance spectroscopy values more in keeping with subjects with Alzheimer's disease than normal controls. The presence of white matter lesions has also been suggested as a cause of cognitive decline, as have neuroendocrine changes such as oestrogen loss and age-related changes in cortisol secretion (O’Brien, 1997).

\section{Classification of mild cognitive disorders}

The previous discussion emphasises that agerelated cognitive changes do occur, that they affect some abilities more than others and, similarly, that they affect some individuals to a much greater extent than others. The question then arises as to the relationship between such age-related changes and more profound changes in cognition recognised in disorders such as Alzheimer's disease, dementia with Lewy bodies, vascular dementia, frontal lobe dementia and others. Should milder degrees of cognitive impairment be classified and, if so, how? The continuum view of cognitive decline with ageing, expounded by some (e.g. Brayne \& Calloway, 1988) suggests that age-related changes and Alzheimer's disease are on a continuum, one end of the continuum representing 'successful' ageing where virtually no age-related change in cognition is seen, the other end of the continuum reflecting rapidly progressive disorders such as Alzheimer's disease. The intermediate stages of cognitive impairment will be seen as occupying an interim position on the spectrum. Such a view would question the usefulness of trying to characterise and label different conditions which merely reflect different points on a continuum. This model can only be supported or refuted by the results of definitive, prospective, longitudinal studies combining cognitive assessment with neuropathological examination, such as the current five-centre Cognitive Function in Ageing study funded by the Medical Research Council. However, clinicians have felt the need to try to classify those with mild cognitive problems for over 35 years, suggesting that there is indeed a clinical requirement for the categorisation of subjects presenting with mild cognitive impairment. Several different diagnostic categories have been proposed and the usefulness of each must be considered in more general terms of the validity of diagnostic constructs.

\section{Categories of diagnosis}

The purpose of a diagnostic label is to describe in shorthand what is known regarding history, symptoms and signs, in order to convey this information to others, particularly with regard to treatment and prognosis. Wulff (1976) suggested four categories of diagnosis: (i) symptom diagnosis; (ii) syndromes; (iii) anatomically defined diseases and (iv) causally defined diseases. While much of medicine deals in anatomically or causally defined diseases, where there is often a specific laboratorybased confirmatory test, psychiatry is generally limited to syndromal diagnosis. The problem of how to validate clinical syndromes in psychiatry is discussed by Kendell (1989). Central to the issue is the demonstration of boundaries or 'points of rarity' from other recognised disorders, in the current case distinction from both successful ageing and dementia would be required. Several distinct strategies have been proposed for establishing the validity of clinical syndromes (Robins \& Guze, 1970; Kendell, 1989) (see Box 1). Proposed ways in which age-related cognitive changes may be classified will be described with reference to their clinical validity.

\section{Benign senescence forgetfulness}

It was Kral $(1958,1962,1978)$ who introduced the terms 'benign' and 'malignant' senescence forgetfulness to describe two distinct types of memory dysfunction which he identified following careful

Box 1. Strategies for validating clinical syndromes (after Robins \& Guze, 1970; Kendell, 1989)

Rigorous identification and description of the syndrome, with regard to both inclusion and exclusion criteria

Demonstration of boundaries or'points of rarity' between the proposed entity and normality and other already accepted diagnoses

Follow-up studies establishing a distinctive course or outcome

Therapeutic trials establishing a distinctive treatment response

Family history studies establishing that the syndrome 'breeds true'

Association with some more fundamental abnormality-histological, psychological, biochemical or molecular 
clinical observations of residents of a Montreal nursing home. Benign senescence forgetfulness (BSF) was characterised by those with mild memory problems with "minor and variable errors in orientation, difficulties in recalling, at times, names and dates of the past which were available at other times". There was no clinical evidence of memory dysfunction. Subjects with malignant forgetfulness had severe memory problems characterised by disorientation, clinical evidence of impairment of recent memory and immediate recall, and sometimes confabulation and nominal dysphasia. Kral validated the distinction between benign and malignant forgetfulness by a four-year follow-up study which demonstrated greater mortality in those with the malignant form.

The syndrome of BSF undoubtedly hit a chord with clinicians and has been used intermittently ever since. However, Kral's studies were based on nursing home residents who were neither randomly selected nor representative of the general population. No objective cognitive tests were used and the absence of specific criteria for BSF made it difficult for others to take this concept forward. Of the limited followup studies performed, BSF essentially appears to be a non-progressive disorder in most cases $\left(\mathrm{O}^{\prime}\right.$ Brien et al, 1992).

\section{Age-associated memory impairment}

Because of the problems associated with the rather vague concept of BSF, a National Institute of Mental Health work group was convened in 1986 to establish specific research criteria "to describe the memory loss that may occur in healthy, elderly individuals in the later decades of life" (Crook et al, 1986). The group proposed use of the term 'ageassociated memory impairment' (AAMI) with the specific diagnostic criteria listed in Box 2.

Age-associated memory impairment represented an important shift from Kral's notion of benign forgetfulness. While Kral sought to distinguish those with BSF from the healthy elderly, AAMI with its criteria of memory loss one standard deviation below that for young adults seeks to define changes associated with ageing, whether 'normal' or otherwise. The concept has been criticised as being over-inclusive and likely to apply to the majority of the over 50s. It is a cross-sectional diagnosis which makes no allowance for decline relative to an individual's performance when young. No specific neuropsychological tests are proposed and it is not clear whether those aged over 50 have to be one standard deviation below the mean on any test, on the majority of tests administered, or on all the tests administered. An eloquent demonstration of some of these problems was provided by Smith et al (1991) who showed that if multiple neuropsychological tests were administered, then cognitive criteria for AAMI could be met by anything from $7 \%$ to $96 \%$ of the healthy elderly. Other problems with the concept of AAMI include the reliance purely on memory impairment, when it is clear that other cognitive changes (e.g. in frontal lobe functioning) can occur with age (Hanninen et al, 1997), as well as reliance on the use of broad screening instruments such as the Mini-Mental State Examination (Folstein et al, 1975) to exclude the absence of dementia.

In common with some other definitions, the inclusion of subjective complaint of poor memory for the diagnosis of AAMI may be problematic. Complaints of poor memory are seen in at least one in four elderly subjects and it is well established that subjective memory complaints and impaired performance on objective psychological tests are poorly correlated (Rabbitt \& Abson, 1990). One explanation for this discrepancy is the strong association between depression and subjective complaints of poor memory (O'Connor et al, 1990).

Although few studies have examined this issue, AAMI appears to be an essentially non-progressive disorder (Hanninen et al, 1995) which would be consistent with its over-inclusive coverage of large numbers of elderly subjects. As yet there is no clear pattern of particular neuroimaging or genetic associations (Palumbo et al, 1997), although, as previously indicated, hippocampal changes may be important. A number of treatment studies have been reported, with mixed results (Riedel \& Jolles, 1996). Overall, the merits of AAMI as a diagnosis remain unclear and some see its main purpose as a label to justify pharmacotherapy for what are essentially

Box 2. Diagnostic criteria for age-associated memory impairment (Crook et al, 1986)

Aged over 50 years

History of gradual memory dysfunction apparent in activities of daily life

Subjective memory complaints substantiated by objective evidence of deficits as measured by performance on a well-standardised memory test at least one standard deviation below the mean established for young adults

Intact global intellectual function

Absence of dementia as determined by a score of $\mathbf{2 4}$ or higher on the Mini-Mental State Examination 
age-related memory changes experienced by the majority of the population.

\section{Ageing-associated cognitive decline}

To try to address some of the problems apparent in the concept of AAMI, a working party supported by the International Psychogeriatric Association and the World Health Organization met to produce a report and propose criteria for a concept they called "aging-associated cognitive decline" (AACD; Levy, 1994). The diagnostic criteria suggested are listed in Box 3. There are several major differences between AACD and AAMI. First, AAMI relies on subjective complaint of memory difficulty, whereas AACD relies on subjective complaint or report from a reliable informant. Criteria for AACD specify that onset needs to be gradual (thus excluding conditions such as stroke) and present for at least six months (excluding other potential reversible disorders). Much more importantly, the disorder is characterised by difficulties in one of five areas: memory and learning; attention and concentration; thinking; language; and visuo-spatial functioning. This broadens the concept from memory to other cognitive domains that are known to be affected by ageing.

Perhaps the central difference, however, is of the performance indicator which is one standard deviation below the mean value for age-matched controls. Thus, AACD seeks to identify those who have some form of cognitive impairment relative to their aged peers, in contrast to AAMI which identifies those who have specifically a memory impairment judged relative to young healthy adults. The work group also identified a number of possible lines of research, including the establishment of reliable age-specific norms for a number of appropriate neuropsychological tests. Detailed studies regarding AACD are awaited and will undoubtedly serve to refine criteria, although the more precise definition, the move away from purely subjective complaint of poor memory and the broadening to include other cognitive domains all have a solid evidence base.

\section{Other categories of mild cognitive decline}

Age-related cognitive decline is included in DSMIV (American Psychiatric Association, 1994) as "an additional condition that may be of focus of clinical attention", although the definition is rather vague (Box 4). It is also possible to diagnose mild cognitive difficulties under the ICD-10 (World Health Organization, 1992) criteria under F06.7 mild cognitive disorder (Box 5), although, perhaps surprisingly, ageing is not listed as a possible cause. Other labels include 'mild dementia', 'questionable dementia', 'very mild cognitive decline', 'limited cognitive disturbance', the 'minimal dementia' category of the Cambridge Mental Disorders of the Elderly Examination (CAMDEX; Roth et al, 1986) and the term 'cognitive impairment no dementia" (CIND; Canadian Study of Health andAgeing, 1994). Many have been the subject of a number of studies, although as there is no consensus regarding the

Box3. Diagnostic criteria for aging-associated cognitive decline (Levy, 1994)

There should be a report by the individual or a reliable informant that cognitive function has declined

Onset of decline must be described as gradual and must have been present for at least six months

The disorder is characterised by difficulties in any one of the following areas: memory and learning; attention and concentration; thinking (e.g. problem-solving, abstraction); language (e.g. comprehension, wordfinding); and visuo-spatial functioning

There is an abnormality of performance on quantitative cognitive assessments (e.g. neuropsychological tests or mental state evaluations) for which age and education norms are available for relatively healthy individuals; performance must be at least one standard deviation below the mean value for the appropriate population

Exclusion criteria - none of the abnormalities listed above is of a sufficient degree for a diagnosis of mild cognitive disorder or dementia to be made; there must be no objective evidence from physical and neurological examination or laboratory tests and no history of cerebral disease, damage, or dysfunction or of systemic physical disorder known to cause cerebral dysfunction; other exclusion criteria as follows: (a) depression, anxiety, or other significant psychiatric disorders that may contribute to observed difficulties; (b) organic amnestic syndrome; (c) delirium; (d) postencephalitic syndrome; (e) postconcussional syndrome; (f) persisting cognitive impairment due to psychoactive substance use or the effects of any centrallyacting drug 
superiority of any single term and none currently fulfils satisfactory criteria for clinical validity, their widespread clinical use cannot be recommended.

\section{Mild cognitive disturbance and early dementia}

Given the high prevalence of dementia in the population ( $5 \%$ of the over- $65 \mathrm{~s}$ and $20 \%$ of the over- $80 \mathrm{~s})$, any category of mild cognitive decline is likely to include at least some patients in the very early stages of dementia. A number of studies have shown that while purely subjective memory complaints do not predict cognitive decline in the majority of cases, objective evidence of impairment relative to agematched controls often does. For example, Reisberg et al (1986) found that two-thirds of subjects with cognitive deficit that fell short of dementia deteriorated over the period of a four-year follow-up. Similarly, O'Connor et al (1991) showed that $50 \%$ of those with a CAMDEX diagnosis of minimal dementia progressed to definite dementia at two-year follow-up. Once again, a category of minimal dementia includes objective evidence of cognitive impairment.

In contrast, Hanninen et al (1995) showed that only $9 \%$ of those with AAMI (essentially subjective complaints only) progressed to clear-cut dementia over a three-year period. The best baseline discriminators between these subjects were impaired memory and verbal fluency. That objective evidence of impairment is the best predictor of dementia was also shown by Howieson et al (1997), who found verbal

Box 4. DSM-IV criteria for age-related cognitive decline (American Psychiatric Association, 1994)

This category can be used when the focus of clinical attention is an objectively identified decline in cognitive functioning consequent to the ageing process that is within normal limits given the person's age. Individuals with this condition may report problems remembering names or appointments or may experience difficulty in solving complex problems. This category should be considered only after it has been determined that the cognitive impairment is not attributable to a specific mental disorder or neurological condition memory impairment at a mean of 2.8 years before onset of clinical dementia in a prospective study.

Other factors which are associated with progression of cognitive deficit include advancing age, possession of apolipoprotein $\varepsilon 4$ (which is an important risk factor for late-onset Alzheimer's disease) and poor physical health. Several factors have been suggested (although not proven) as protective against the development of Alzheimer's disease including higher education and intelligence, use of hormone replacement therapy and use of antiinflammatories. Whether similar factors may be protective against age-related cognitive changes is unknown.

Box 5. ICD-10 criteria for F06.7, mild cognitive disorder (World Health Organization, 1992)

This disorder may precede, accompany, or follow a wide variety of infections and physical disorders, both cerebral and systemic (including HIV infection). Direct neurological evidence of cerebral involvement is not necessarily present, but there may nevertheless be distress and interference with usual activities. The boundaries of this category are still to be firmly established. When associated with a physical disorder from which the patient recovers, mild cognitive disorder does not last for more than a few additional weeks. This diagnosis should not be made if the condition is clearly attributable to a mental or behavioural disorder classified in any of the remaining blocks in ICD-10

\section{Diagnostic guidelines}

The main feature is a decline in cognitive performance. This may include memory impairment, learning or concentration difficulties. Objective tests usually indicate abnormality. The symptoms are such that a diagnosis of dementia (F00-F003), organic amnestic syndrome (F04) or delirium (F05.-) cannot be made

\section{Differential diagnosis}

The disorder can be differentiated from postencephalitic syndrome (F07.1) and postconcussional syndrome (F07.2) by its different aetiology, more restricted range of generally milder symptoms, and usually shorter duration 


\section{Should age-associated cognitive decline be treated?}

The treatment of subjective memory complaints in older people is usually limited to the physician giving reassurance that no evidence of dementia can be found. This should only be after full and careful assessment, investigation for common disorders associated with mild memory impairment (e.g. depression, infections, metabolic and endocrine disorders) and the administration of objective tests of cognitive function. More specialist assessment, such as that provided through a hospital memory clinic, may also be required, particularly if no other cause for memory complaints can readily be identified or if there is any objective evidence of impairment. In other cases intervention should be aimed at the identification and treatment of depression and ensuring optimum treatment of co-

Box 6. Key teaching points

Subjective complaint of poor memory is common in the elderly

Such complaints have a stronger association with depression than dementia

Other cognitive functions show decline as well as memory

Complaints of impaired cognition should be taken seriously and full and careful assessment is needed to exclude depression and dementia and other conditions that may affect cognition (e.g. side-effects of medication, metabolic and endocrine disturbance, infections, neoplasms)

Referral to local old age psychiatry services or a specialist memory clinic may be indicated

Those with subjective memory problems, in the absence of objective evidence of impairment, do not usually show progression to dementia

Currently licensed treatments for Alzheimer's disease are not indicated for those with age-associated memory impairment or age-related cognitive decline

The nosological status of mild cognitive disorders remains unclear; further refinement with careful study of clinical validity is required existing physical disorders which may contribute to memory loss. The place for pharmacological treatments is unclear. Cholinesterase inhibitors have recently been introduced for Alzheimer's disease and show definite, albeit relatively modest, benefit. The question then arises as to whether those with mild cognitive impairment should be treated. There are two issues, the first is whether cognitive enhancing compounds actually work in elderly subjects without dementia, and the second is, if so, whether this use for such compounds should be advocated. (Please see Box 6 for a list of key teaching points.)

There are a number of studies which have addressed the first issue and there is evidence that some compounds may be effective. For example, a review by Riedel \& Jolles (1996) found many studies in which cognitive enhancing properties of substances could be reliably demonstrated, with many studies including substances already in wide use (caffeine and nicotine) as well as other compounds. However, they concluded that there was no cognitive enhancer that had reliably and repeatedly been demonstrated to be efficacious for the treatment of age-associated cognitive decline. It is likely that this situation, with the introduction of more selective and specific compounds, may change in the future. If so, the complex and wider ethical issue of whether such 'normal' cognitive impairment should be treated will emerge. One view is that, like age-related changes in vision and hearing, if effective and simple treatments for age-related cognitive changes become available they should be used. The opposite view is that the treatment of such conditions is more akin to the use of anabolic steroids to improve muscle performance and should not be advocated. To some extent the answer will surely depend on the availability, cost and tolerability of any suggested drug. Consensus is likely to be different with regard to a specific pharmacological compound with a number of side-effects that is highly expensive, compared with a relatively innocuous and cheap vitamin pill taken daily.

One should also not ignore possible psychological approaches. The most successful strategies have been those adapted from use by all of us to aid memory, such as using a notice board, making lists, using extended diaries and the use of mnemonics. Such techniques rely heavily on the use of environmental cues to aid memory. Alternative approaches are to look at theoretical memory processes and how they may be manipulated, for example, by the use of verbal elaborational imagery to link the stimulus to the response the subject is required to learn. While such techniques have proved successful in improving performance in the laboratory, their general applicability may be more limited. 


\section{Conclusions}

There is little doubt that changes in cognition occur in most of us as we age. Although much progress has been made in recent years in the classification of cognitive decline that falls short of dementia, there is still no universally accepted method of describing these syndromes. They are inadequately dealt with in ICD-10 and DSM-IV. Early definitions lacked standardisation and objective measurement, and placed the whole emphasis on memory when it was known that other cognitive abilities decline. More recent descriptions (e.g. AACD) appear to represent a sensible step to define clear groups who are impaired relative to their peers. Further refinement and studies on the clinical validity of these and other criteria are required. The neurobiological basis of age-related cognitive changes remains unclear, although some may be related to degenerative changes and tangle formation in hippocampus and associated regions.

No clearly effective treatment for age-related cognitive changes currently exists and, even if one did, there would be practical, ethical and economic issues regarding when and how and to whom such a treatment should be delivered. Clinically, the greatest value may be in the identification of subjects in an early or 'preclinical' stage of dementia. This will certainly be the case if neuroprotective therapies become available. Such groups may be identified by virtue of their age, genotype and the meeting of particular criteria for a condition known to be at high risk of progression to dementia.

\section{References}

American Psychiatric Association (1994) Diagnostic and Statistical Manual of Mental Disorders (4th edn) (DSM-IV). Washington, DC: APA.

Anderson, J. M., Hubbard, G. R., Coghill, G. R., et al (1983) The effect of advanced old age on neurone content of the cerebral cortex; observations with an automatic image analyzer point counting method. Journal of Neurological Sciences, 58, 235-46.

Birren, J. E., Woods, A. M. \& Williams, M. V. (1979) Speed of behaviour as an indicator of age changes and the integrity of the nervous system. In Brain Function in Old Age (eds F. Hoffmeister \& C. Muller), pp. 10-14. Berlin: SpringerVerlag.

Botwinick, J. (1977) Intellectual abilities. In Handbook of the Psychology of Aging (eds J. E. Birren \& K. W. Schaie), pp. 580-605. New York: Van Nostrand Reinhold.

Brayne, C. \& Calloway, P. (1988) Normal ageing, impaired cognitive function, and senile dementia of the Alzheimer's type: a continuum? Lancet, $i, 1265-1267$.

Brody, H. (1955) Organisation of the cerebral cortex: III. A study of aging in the human cerebral cortex. Journal of Comparative Neurology, 102, 511-56.
Buell, S. J. \& Coleman, P. D. (1979) Dendritic growth in the aged human brain and failure of growth in senile dementia. Science, 206, 854-856.

Canadian Study of Health and Ageing (1994) Risk factors for Alzheimer's disease in Canada. Neurology, 44, 20732080.

Craik, F. I. M. \& Byrd, M. (1982) Ageing and cognitive deficits; the role of attentional resources. In Ageing and the Cognitive Processes (eds F. I. M. Craik \& S. E. Trehub), pp. 191-211. New York: Plenum Press.

Crook, T., Bartus, R. T., Ferris, S. H., et al (1986) Ageassociated memory impairment: proposed diagnostic criteria and measures of clinical change - report of a National Institute of Mental Health work group. Developmental Neuropsychology, 2, 261-76.

Dal Forno, G. \& Kawas, C. H. (1995) Cognitive problems in the elderly. Current Opinion in Neurology, 8, 256-261.

Folstein, M. F., Folstein, S. E. \& McHugh, P. R. (1975) 'MiniMental State': a practical method for grading the cognitive state of patients for the clinician. Journal of Psychiatric Research, 12, 189-198.

Giannakopoulos, P., Hof, P. R., Michel, J. P., et al (1997) Cerebral cortex pathology in ageing and Alzheimer's disease: a quantitative survey of large hospital-based geriatric and psychiatric cohorts. Brain Research - Brain Research Reviews, 25, 217-245.

Hanninen, T., Hallikainen, M., Koivisto, K., et al (1995) A follow-up study of age-associated memory impairment: neuropsychological predictors of dementia. Journal of the American Geriatrics Society, 43, 1007-1015.

-, - \& - (1997) Decline of frontal lobe functions in subjects with age-associated memory. Neurology, 48, 148153.

Hof, P. R., Glannakopoulos, P. \& Bouras, C. (1996) The neuropathological changes associated with normal brain ageing. Histology \& Histopathology, 11, 1075-1088.

Horn, J. L. \& Cattell, R. B. (1967) Age differences in fluid and crystallised intelligence. Acta Psychologia, 26, 107129.

Howieson, D. B., Dame, A., Camicioli, R., et al (1997) Cognitive markers preceding Alzheimer's dementia in the healthiest oldest old. Journal of the American Geriatrics Society, 45, 584-589.

Kendell, R. E. (1989) Clinical validity. Psychological Medicine, $19,45-55$.

Kral, V. A. (1958) Neuropsychiatric observations in an old people's home. Journal of Gerontology, 13, 169-176.

- (1962) Senescent forgetfulness: benign and malignant. Canadian Medical Association Journal, 86, 257-260.

- (1978) Benign sensecent forgetfulness. In Alzheimer's Disease: Senile Dementia and Related Disorders (eds R. Katzman., B. D. Terry., K. L. Bick), pp. 47-51. New York: Raven Press.

Laakso, M. P., Soininen, H., Partanen, K., et al (1998) MRI of the hippocampus in Alzheimer's disease: sensitivity, specificity, and analysis of the incorrectly classified subjects. Neurobiology of Ageing, 19, 23-31.

Levy, R. (1994) Aging-associated cognitive decline. International Psychogeriatrics, 6, 63.

Nelson, A. E. \& Dannefer, D. (1992) Aged heterogeneity: fact or fiction? The fate of diversity in gerontololgical research. Gerontologist, 32, 17-23.

O'Brien, J. T. (1997) The 'glucocorticoid cascade' hypothesis in man. Prolonged stress may cause permanent brain damage. British Journal of Psychiatry, 170, 199-201.

— \& Beats, B. (1994) Benign sensecent forgetfulness and age-associated memory impairment. In Dementia (eds R. Levy \& A. Burns), pp. 295-308. London: Chapman \& Hall. - - Hill, K., et al (1992) Do subjective memory complaints precede dementia? A 3 year follow up of patients presenting with supposed 'benign sensecent forgetfulness'. International Journal of Geriatric Psychiatry, 7, 481-486.

O'Connor, D. W., Politt, P., Roth, M., et al (1990) Memory complaints and impairment in normal, depressed and demented elderly persons identified in a community survey. Archives of General Psychiatry, 47, 224-227. 
,-- Jones, B. J., et al (1991) Continued clinical validation of dementia diagnosed in the community using the Cambridge Mental Disorders of the Elderly Examination. Acta Psychiatrica Scandinavica, 83, 41-45.

Palumbo, B., Parnetti, L., Nocentini, G., et al (1997) Apolipoprotein-E genotype in normal ageing, ageassociated memory, Alzheimer's disease and vascular dementia patients. Neuroscience Letters, 231, 59-61.

Parnetti, L., Lowenthal, D. T., Presciutti, O., et al (1996) IHMRS, MRI-based hippocampal volumetry, and $99 \mathrm{mTc}$ HMPAO-SPECT in normal ageing, age-associated memory impairment, and probable Alzheimer's disease. Journal of the American Geriatrics Society, 44, 133-138.

Rabbitt, P. \& Abson, V. (1990) 'Lost and found'; Some logical and methodological limitations of self-report questionnaires as tools to study cognitive ageing. British Journal of Psychology, 81, 1-16.

Reisberg, B., Ferris, S. H., Shulman, E., et al (1986) Longitudinal course of normal ageing and progressive dementia of the Alzheimer's type; a prospective study of 106 subjects over 3.6 year mean interval. Progress in Neuropsychopharmacology and Biological Psychiatry, 10, 571-578.

Riedel, W. J. \& Jolles, J. (1996) Cognition enhancers in agerelated cognitive decline. Drugs and Ageing, 8, 245-274.

Ritchie, K. (1995) Mental status examination of an exceptional case of longevity. J. C. aged 118 years. British Journal of Psychiatry, 166, 229-235.

Robins, E. \& Guze, S. B. (1970) Establishment of diagnostic validity in psychiatric illness; its application to schizophrenia. American Journal of Psychiatry, 126, 983-987.

Roth, M., Tym, E., Mountjoy, C., et al (1986) CAMDEX; a standardised instrument for the diagnosis of mental disorder in the elderly with special reference to the early detection of dementia. British Journal of Psychiatry, 149, 698-709.

Sattler, J. M. (1982) Age effects on Wechsler Adult Intelligence Scale - revised tests. Journal of Consultative Clinical Psychology, 50, 785-786.

Schaie, K. W. (1983) The Seattle longitudinal study: a 21year exploration of psychometric intelligence in adulthood. In Longitudinal Studies of Adult Psychological Development (ed. K. E. Schaie), pp. 64-138. New York: Guildford Press.

- Maitland, S. B., Willis, S. L., et al (1998) Longitudinal invariance of adult psychometric ability factor structures across 7 years. Psychology and Ageing, 13, 8-20.

Smith, G., Ivnik, R. J., Peterson, R. C., et al (1991) Ageassociated memory impairment diagnoses: problems of reliability and concerns for terminology. Psychology and Aging, 6, 551-558.

Terry, R. D., DeTeresa, R. \& Hansen, L. A. (1987) Neocortical cell counts in normal human aging. Annals of Neurology, 21, 530-539.

Tomlinson, B. E. \& Henderson, G. (1976) Some quantitative cerebral findings in normal and demented old people. In Neurobiology of Ageing (eds R. D. Terry \& S. Gershon), pp. 183-204. New York: Raven Press.

World Health Organization (1992) International Classification of Diseases and Related Health Problems (10th revision) (ICD10). Geneva: WHO.

Wulff, H. R. (1976) Rational Diagnosis and Treatment. Oxford: Blackwell Scientific.

\section{Multiple choice questions}

1. Characteristic features of cognitive changes with ageing include:

a no change in fluid intelligence

b a decline in episodic memory

c a decline in verbal abilities after the age of $\mathbf{3 0}$ d disinhibition

e no change in cognitive abilities apart from memory.

2. Age-associated memory impairment:

$a$ is the same as benign senescent forgetfulness

b is the same as minimal dementia

c affects approximately $5 \%$ of the over- $65 \mathrm{~s}$

d shows a high rate of progression to dementia

e is included within DSM-IV and ICD-10.

3. Subjective complaints of poor memory in the elderly:

a are common

b usually indicate the presence of an early dementia

c are necessary for a diagnosis of age-associated memory impairment

$\mathrm{d}$ may indicate the presence of depression

e may be due to physical disorders such as hypothyroidism.

4. The following are predictors of cognitive decline: a advancing age

$b$ possession of certain genetic risk factors (e.g. Apo \&4)

c history of use of anti-inflammatory drugs

d subjective memory complaints in the absence of objective impairment

e physical ill health.

5. Established management for those presenting with age-related cognitive decline includes:

a full psychiatric assessment

b use of cholinesterase inhibitors

c advice regarding mnemonic aids

d screening for depression

e reassurance.
MCQ answers

$\begin{array}{llllllllll}\text { 1 } & & \text { 2 } & & 3 & & 4 & & 5 & \\ \text { a } & \text { F } & \text { a } & \text { F } & \text { a } & \text { T } & \text { a } & \text { T } & \text { a } & \text { T } \\ \text { b } & \text { T } & \text { b } & \text { F } & \text { b } & \text { F } & \text { b } & \text { T } & \text { b } & \text { F } \\ \text { c } & \text { F } & \text { c } & \text { F } & \text { c } & \text { T } & \text { c } & \text { F } & \text { c } & \text { T } \\ \text { d } & \text { F } & \text { d } & \text { F } & \text { d } & \text { T } & \text { d } & \text { F } & \text { d } & \text { T } \\ \text { e } & \text { F } & \text { e } & \text { F } & \text { e } & \text { T } & \text { e } & \text { T } & \text { e } & \text { T }\end{array}$

